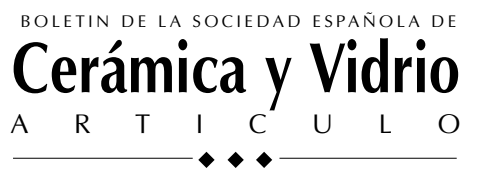

\title{
Desarrollo y caracterización de varistores de óxido de cinc
}

\author{
P. ATTOLINI', E.F. AGLIETTI', A.N. SCIAN² \\ ${ }^{1}$ FAPA S.A. - Argentina. \\ ${ }^{2}$ CETMIC (CIC-CONICET-UNLP)- Cno. Centenario y 506, CC49 (1897), Gonnet, Pcia. Bs. As. Argentina.
}

\begin{abstract}
En el presente trabajo se comparan el comportamiento eléctrico y las características estructurales (DRX), fisicoquímicas y microestructurales (Microscopía óptica, electrónica y microsonda) de cuatro varistores comerciales de distinta procedencia con otros tres desarrollados como prototipos. El análisis comparativo de los resultados obtenidos para todos los varistores estudiados permitió conocer las causas del bajo rendimiento de los prototipos desarrollados. Un mayor control en las etapas de procesamiento de las materias primas, del prensado y del tratamiento térmico, conjuntamente con un aumento en la composición de $\mathrm{Sb}_{2} \mathrm{O}_{3}$ en la formulación del prototipo denominado $\mathrm{M} 2$ podría conducir a un varistor con buenas prestaciones de servicio.
\end{abstract}

\section{Palabras clave: varistores, óxido de zinc, desarrollo y caracterización de varistores}

\section{Developemet and characterization of zinc oxide varistors}

Abstract. In this paper the electric behaviour and other structural, physicochemical and micro structural characteristics of four commercial varistors of different makers were studied and compared with other three developed prototypes. The comparative results permitted to know the causes of the prototypes low performance, concluding that with a better control of the raw materials processing, the pre-forming and the thermal treatment, in conjunction with an increase of the $\mathrm{Sb}_{2} \mathrm{O}_{3}$ quantities in the prototype $\mathrm{M} 2$, a varistor with a good performance may be produced.

Keywords: varistors, zinc oxide, development and charaterization of varistors

Trabajo presentado en la III Reunión Iberoamericana en Materiales Electrocerámicos. (México, Abril 2000).

\section{INTRODUCCIÓN}

Los circuitos eléctricos y electrónicos están sujetos a severos impulsos de voltaje transitorios generados por encendidos o descargas electrostáticas. Para tal fin se han diseñado elementos protectores que actúan como supresores de estos transitorios. Existen varios tipos de supresores siendo los más usados en la actualidad los denominados varistores de óxido de zinc. El varistor de $\mathrm{ZnO}$ tiene un comportamiento no lineal para la curva voltaje-corriente y por lo tanto puede soportar una amplia variación de corriente en un rango estrecho de voltaje. Este fenómeno es denominado efecto varistor y fue descubierto por Matsuoka en 1968 (1). A bajos voltajes el varistor opera como en circuito abierto, pero al alcanzar el voltaje de conmutación actúa como componente protector.

La propiedad no-ohmica está promovida por la presencia de una segunda fase en los bordes de grano del $\mathrm{ZnO}$. La propiedad no lineal tensión-corriente se evalúa por un coeficiente de no-linealidad $\alpha$, siendo mejor cuanto mayor es dicho valor. Este valor corresponde a la zona de ruptura y corresponde a la acción esencial del varistor. La estructura básica está constituída por granos de $\mathrm{ZnO}$ dopados y una región intergranular rica en $\mathrm{Bi}^{+3}$. Se utilizan además otros óxidos como aditivos para mejorar las propiedades del varistor (2-4).
Los óxidos utilizados en los varistores de $\mathrm{ZnO}$ han sido divididos en grupos de acuerdo a su función (2). $\mathrm{El} \mathrm{Bi}_{2} \mathrm{O}_{3}$ es parte de la estructura básica, otros contribuyen a las propiedades no-ohmicas tales como $\mathrm{MnO}_{2^{\prime}} \mathrm{Al}_{2} \mathrm{O}_{3^{\prime}} \operatorname{In}_{2} \mathrm{O}_{3^{\prime}}$ y otros como $\mathrm{Sb}_{2} \mathrm{O}_{3}, \mathrm{Cr}_{2} \mathrm{O}_{3}$ y $\mathrm{NiO}$ contribuyen a la estabilidad. La composición mas utilizada contiene además de $\mathrm{ZnO}, \mathrm{Bi}_{2} \mathrm{O}_{3^{\prime}}$ $\mathrm{Sb}_{2} \mathrm{O}_{3}, \mathrm{CoO}, \mathrm{Cr}_{2} \mathrm{O}_{3}$ y $\mathrm{MnO}_{2}$ (5). Las fases secundarias predominantes, luego del proceso de sinterización son: $\mathrm{Bi}_{2} \mathrm{O}_{3}$, $\mathrm{Zn}_{7} \mathrm{Sb}_{2} \mathrm{O}_{12}$ (con estructura de espinela) y $\mathrm{Zn}_{2} \mathrm{Bi}_{3} \mathrm{Sb}_{3} \mathrm{O}_{14}$ (con estructura pirocloro), aunque la función de muchos aditivos y sus productos de reacción en las propiedades no lineales y de envejecimiento no está totalmente dilucidada (6).

$\mathrm{El} \mathrm{Sb}_{2} \mathrm{O}_{3}$ contribuye a una estabilidad de la fase espinela e impide el crecimiento de grano, el $\mathrm{MnO}$ proveniente de la descomposición del $\mathrm{MnO}_{2}$ agregado como dopante modifica en cambio el valor de $\alpha$. Se suele indicar como composición básica para el varistor la constituida por $\mathrm{ZnO}$ (98 moles \%) y $\mathrm{Bi}_{2} \mathrm{O}_{3^{\prime}}, \mathrm{Sb}_{2} \mathrm{O}_{3^{\prime}} \mathrm{CoO}$ y $\mathrm{MnO}_{2}$ con contenidos del $0,5 \%$ en moles para cada uno de ellos. De todas formas esta es una composición indicativa ya que el efecto de estos aditivos está fuertemente condicionado al procesamiento y las condiciones de sinterización del material. 
TABLA 1. COMPOSICIÓN DE LOS VARISTORES PROTOTIPO M1, M2 Y M3 -PARTES EN MOLES DE LOS ÓXIDOS UTILIZADOS EN LA PREPARACIÓN DE LA MEZCLA CRUDA.

\begin{tabular}{|lcccccccc|}
\hline \multicolumn{7}{|c|}{ Partes en Moles } \\
\hline & $\mathbf{Z n O}$ & $\mathbf{B i}_{\mathbf{2}} \mathbf{O}_{3}$ & $\mathbf{C o}_{\mathbf{2}} \mathbf{O}_{\mathbf{3}}$ & $\mathbf{M n O} \mathbf{M}_{\mathbf{2}}$ & $\mathbf{S b}_{\mathbf{2}} \mathbf{O}_{3}$ & $\mathbf{C r}_{\mathbf{2}} \mathbf{O}_{3}$ & $\mathbf{N i O}$ & $\mathbf{M g O}$ \\
\hline $\mathbf{M 1}$ & 95 & 0,50 & 0,50 & 0,50 & 1,00 & 0,50 & 1,00 & 0,50 \\
$\mathbf{M 2}$ & 95 & 0,55 & 0,55 & 0,55 & 1,10 & 0,55 & 1,10 & 0,55 \\
$\mathbf{M 3}$ & 95 & 0,55 & 0,55 & 0,60 & 1,10 & 0,55 & 1,10 & 0,55 \\
\hline
\end{tabular}

\section{PROCEDIMIENTO EXPERIMENTAL}

En la Tabla 1 se muestran las composiciones de los tres varistores prototipo preparados (M1, M2 y M3). El varistor M2 se elaboró con un $10 \%$ mas de óxidos dopantes respecto al M1; siendo la formulación del M3 igual que el M2 pero con un $20 \%$ en exceso de $\mathrm{MnO}_{2}$ respecto del M1. El diámetro de partícula medio del $\mathrm{ZnO}$ fue de aproximadamente $6 \mu \mathrm{m}$, mientras que para los otros óxidos fue de unos $2 \mu \mathrm{m}$.

En el proceso de obtención se siguieron los siguientes pasos:

1. Molienda húmeda (agua desionizada) en molino de bolas de todos los óxidos dopantes con excepción del $\mathrm{Bi}_{2} \mathrm{O}_{3^{\prime}}$ seguida de secado y calcinación de los mismos a $800^{\circ} \mathrm{C}$ en horno eléctrico durante $1 \mathrm{~h}$.

2. Calcinación del polvo de $\mathrm{ZnO}$ durante $1 \mathrm{~h}$ a $900{ }^{\circ} \mathrm{C}$ (eliminación de posibles impurezas orgánicas en esta materia prima) y posterior molienda en húmedo conjuntamente con el $\mathrm{Bi}_{2} \mathrm{O}_{3}$

3. Adición de los óxidos dopantes previamente molidos a la carga del molino que contiene el $\mathrm{ZnO}+\mathrm{Bi}_{2} \mathrm{O}_{3}$ y molienda conjunta.

4. Secado de la mezcla total a $110^{\circ} \mathrm{C}$ hasta peso constante. Granulación del material seco hasta pasante malla 18 ASTM (1,00 mm).

5. Prensado uniaxial en seco a $55 \mathrm{MPa}$-formato cilíndrico-.

6. Calcinación en horno eléctrico con curva controlada - 1 h a temperatura máxima de $1250{ }^{\circ} \mathrm{C}$-.

7. Esmaltado de superficies laterales y tratamiento térmico a $1200{ }^{\circ} \mathrm{C}$ durante $1 \mathrm{~h}$.

8. Aplicación de pintura conductora de grafito a las superficies circulares de contacto.

Las piezas así obtenidas -diámetro $34 \mathrm{~mm}$ y altura $29 \mathrm{~mm}$ fueron sometidas a ensayos eléctricos: medición de la corriente de fuga característica y medición de la zona de post ruptura -alta densidad de corriente-.

Por otro lado se analizaron por Difracción de Rayos X las fases presentes y en especial la posición del espaciado del pico máximo de la espinela $\mathrm{Sn}_{7} \mathrm{Sb}_{2} \mathrm{O}_{12}$ - plano 311-, el cual mostró poseer una correlación con respecto a las propiedades medidas para el efecto varistor.

Para la determinación del espaciado mencionado $(\mathrm{hkl}=$ 311) se utilizó un barrido en pasos incrementales de $0,02^{\circ}$ de $2 \theta$ para radiación de $\mathrm{Cu} \mathrm{K} \alpha_{1}$, lo que permitió tener un error de \pm 0,0004 $\AA$ en los valores medidos. El goniómetro se alineó con una pastilla de silicio.

Se realizaron observaciones con microscopía óptica para evaluar macrodefectos y además se utilizó la microscopía electrónica para observar micro-estructuras, texturas y porosidades de muestras pulidas, parcialmente atacadas y atacadas con ácido acético al $10 \% \mathrm{v} / \mathrm{v}$. Se analizaron con microsonda electrónica las composiciones químicas de los bordes de grano sobre muestras pulidas y observadas con una magnificación de 3000X. La sonda con resolución aproximada de $1 \mu \mathrm{m}^{3}$ se ubicó en los bordes de grano que poseían mayor definición de imagen.

Todos los ensayos de caracterización antes mencionados se realizaron además sobre materiales comerciales de distinta procedencia, identificados como: M4 (USA), M5 (CE), M6 (USA) y B (Brasil). La información obtenida de estos últimos se correlacionó con la obtenida para los varistores desarrollados como prototipos.

Para evitar distorsiones en la interpretación de los resultados debida a las diferencias geométricas de longitud y diámetro de las muestras de referencia entre sí y con respecto a la de los prototipos, los valores de las mediciones eléctricas se refirieron a "tensión/longitud" y "corriente/área".

\section{RESULTADOS Y DISCUSIÓN}

En la Figura 1 se visualizan las distintas curvas de corriente de fuga $-\mathrm{kV} / \mathrm{mm}$ vs. $\mathrm{mA} / \mathrm{cm}^{2}$ - obtenidas para los distintos varistores a $1 ; 5$ y $10 \mathrm{~mA}$ y a $50 \mathrm{~Hz}$.

Se observa que los varistores en desarrollo -M1; M2 y M3no solo poseen tensiones de conmutación mucho mas bajas que los materiales comerciales, sino que además poseen un mayor incremento en los valores de campo eléctrico para un mismo intervalo de flujo de corriente. Para el prototipo M2 la tensión de referencia fue de $2,7 \mathrm{kV}$ eficaz a una intensidad de referencia máxima de $5 \mathrm{~mA}$, siendo el $\mathrm{C}_{0}$ (VMOC - Voltaje máximo de operación continua ) de $2,22 \mathrm{kV}$.

La observación del gráfico marca además un ordenamiento general en cuanto a las bondades de los varistores analizados, ubicándose en orden decreciente frente a esta propiedad de la siguiente forma: M4; M5; M6; B; M2; M3; M1.

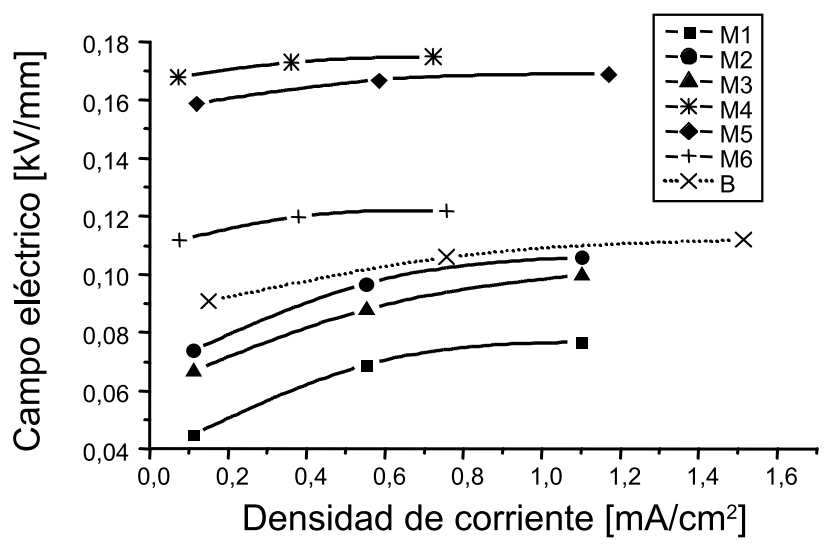

Fig. 1: Campo eléctrico $[\mathrm{kV} / \mathrm{mm}]$ vs. densidad de corriente $\left[\mathrm{mA} / \mathrm{cm}^{2}\right]$ para los distintos varistores estudiados.

En la Figura 2, se representa la posición del pico máximo de la espinela $\mathrm{Zn}_{7} \mathrm{Sb}_{2} \mathrm{O}_{12}(\mathrm{hkl}=311)$, notándose que ninguno de los varistores analizados posee el mismo espaciado. Lo que si puede observarse es que si se ordenan en orden decreciente de

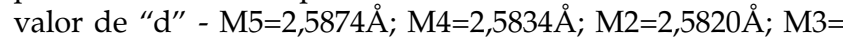
$\mathrm{M} 1=2.5794 \AA$ - el orden que presentan es casi similar al observado para la propiedad de corriente de fuga; las excepciones son el orden M4-M5 en y la igualdad M1-M3, pero de todas formas coinciden en mostrar una tendencia a que cuanto mejor 
se portan eléctricamente es cuanto mayor es el valor del espaciado medido. No se observaron desviaciones en la posición de los picos de $\mathrm{ZnO}$.

Obsérvese que el varistor M1 mejoras sus propiedades eléctricas cuando se modifica su composición de aditivos llevándolo a la composición del varistor $\mathrm{M} 2$, el que a su vez tiende a acercar el espaciado mencionado a los valores medidos para los mejores varistores comerciales; mientras que cuando a la formulación M2 se le agrega mayor cantidad de $\mathrm{MnO}_{2}$ (varistor $\mathrm{M} 3$ ), las propiedades eléctricas se desmejoran a niveles iguales o peores que las del M1. Si bien la característica del espaciado medido no es directa-mentamente la responsable de que un varistor sea bueno o malo, sí podría estar indicando que el despalzamiento de este pico mostraría una descompensación de alguno de los aditivos en la composición global, lo que haría que el equilibrio de fases presentes entre los granos de $\mathrm{ZnO}$, y la composición de una o mas de esas fases - presencia de defectos por ejemplo - estuviera en valores aceptables para que se manifieste un buen efecto varistor o no.

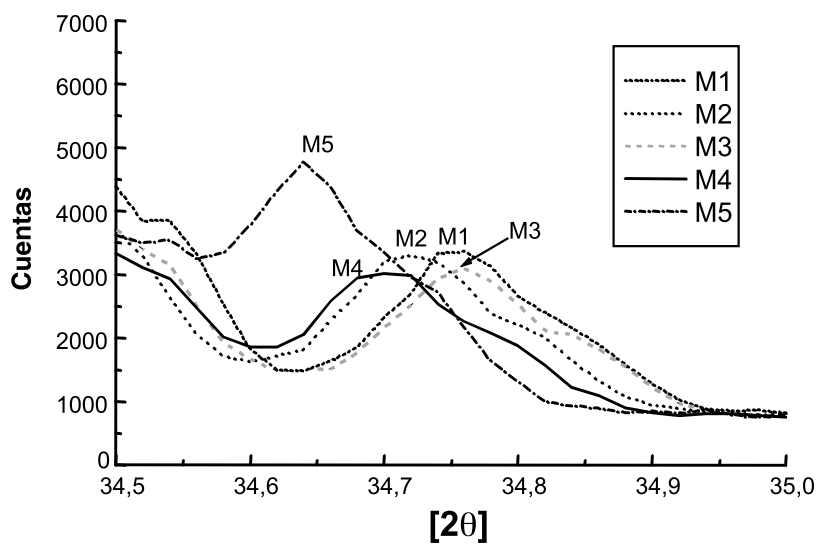

Fig. 2. Difractograma de RX correspondiente a las muestras analizadas en la zona del pico de máxima intensidad de la espinela.

En la región de ruptura por otra parte - zona de la Figura 1 superior a $0.1 \mathrm{~mA} / \mathrm{cm}^{2}$ - es donde se pone de manifiesto el llamado "efecto varistor", o sea que la corriente adopta un comportamiento no lineal en función del voltaje aplicado, pudiendo este fenómeno ser representado por la siguiente ecuación:

$$
\mathrm{I}=\mathrm{KV}^{\alpha}
$$

donde $\alpha$ es el coeficiente no lineal ( $>1)$ y K una constante que depende de la geometría. A mayor valor de $\alpha$ mejor será el varistor pues ofrecerá mejor protección. En la Figura 3 se representan los valores de $\log$ I en función de $\log \mathrm{V}$ para iguales condiciones geométricas de los distintos varistores estudiados, pudiéndose observar que los mayores valores de pendiente corresponden a los representados en el sector derecho del gráfico y que el ordenamiento en orden de calidad decreciente corresponde al mismo que se dio para las corrientes de fuga. Obsérvese en la Figura 3 que la muestra M3 (con mayor contenido de $\mathrm{MnO}$ ) posee un abrupto cambio de pendiente (baja el valor de $\alpha$ respecto a los otros varistores en el intervalo superior, $\log$ I entre 0,699 y 1 ), confirmando los datos bibliográficos que mencionan que este óxido es un modificador del valor de $\alpha$. En este caso no solo lo modifica sino que lo hace en sentido de desmejorar el efecto varistor, tal como se había observado en los ensayos realizados.

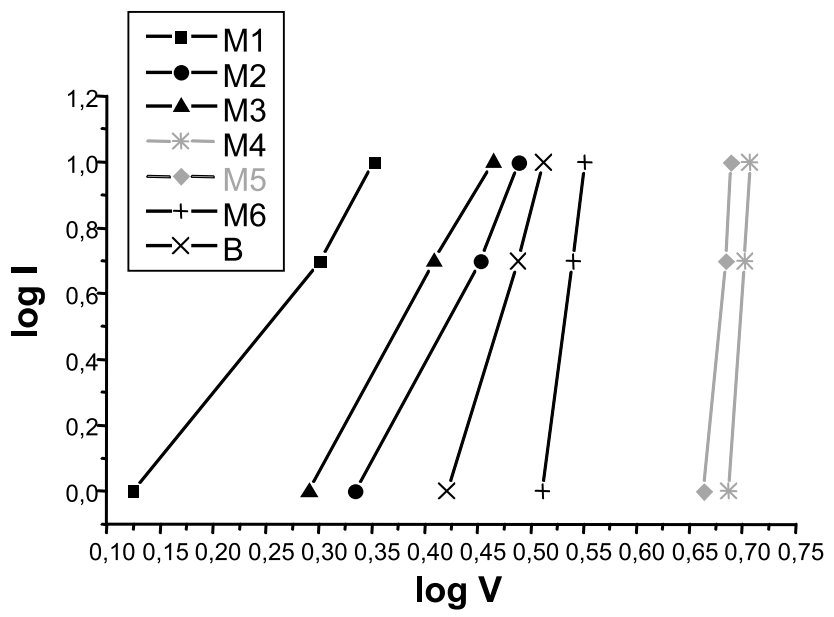

Fig. 3. $\log \mathrm{I}$ vs. $\log \mathrm{V}$ de los distintos varistores en la zona de ruptura. La pendiente de las rectas corresponde al valor de $\alpha$.

Se toma como referencia de valor de ruptura al valor del voltaje de un varistor cuando circula por el una corriente de $1 \mathrm{~mA}$. Teniendo en cuenta entonces los valores medidos para los distintos varistores en iguales condiciones geométricas, se puede aplicar a todos ellos la ecuación válida para los varistores, que según Lagrange (5) es:

$$
\mathrm{V}_{1 \mathrm{~mA}}=3 \mathrm{E} / \mathrm{d}=3 \mathrm{n}
$$

donde $\mathrm{E}$ es el espesor del material, $d$ el tamaño de grano, $\mathrm{V}_{1 \mathrm{~mA}}$ el voltaje de ruptura y $n$ el número de barreras en la dirección del campo eléctrico, observándose que cuanto mas pequeño es el $d$ existe mayor número de barreras y en consecuencia el varistor tendrá una respuesta mejor.

Despejando $d$ de la ecuación [2] y utilizando los valores de E y V para 1, 5, y $10 \mathrm{~mA}$ medidos sobre los materiales en ensayo se construyó la Tabla 2, la cual muestra que los valores calculados de $d$ a partir de los datos experimentales marcan un orden que coincide con el orden asignado al medir las corrientes de fuga y al calcular la pendiente $\alpha$.

Debe mencionarse que si bien los valores de $d$ (tamaño de grano) podrían medirse experimentalmente a través de un mapeo de las microfotografías, la determinación de este valor a través de la medición de $\mathrm{V}_{1 \mathrm{~mA}}$ con la ecuación [2] arroja un valor medio de los granos que están trabajando en el varistor como tal.

Por otro lado si bien la ecuación [2] es válida para el voltaje medido cuando circula una corriente de $1 \mathrm{~mA}$, al hacer además el cálculo para cuando circulan corrientes de 5 y $10 \mathrm{~mA}$ y comparando los valores obtenidos para los distintos varistores -Tabla 2-, se puede inferir que las muestras que presentan el mejor efecto varistor - M4, M5 y M6- muestran un valor casi constante del valor de $d$ calculado, independientemente de la intensidad de corriente que se hace circular por él, mientras que cuanto peor es la muestra ensayada se observa una gran variación de los valores calculados de $d$, correspondiéndose a un ordenamiento similar de propiedades varistor al ya mencionado. 
En la Figura 4 se representan los valores de campo eléctrico en función de densidad de corriente pero para altos valores de ésta última.

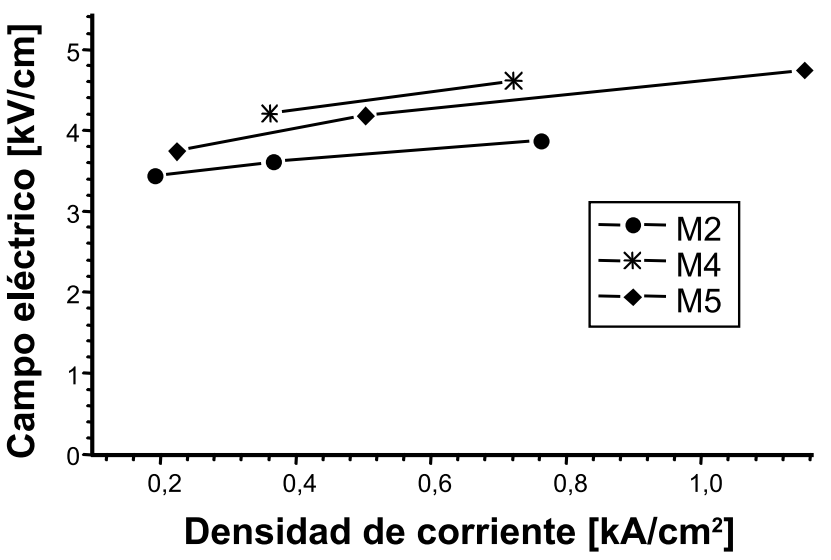

Fig. 4. Curvas de campo eléctrico vs. densidad de corriente para los varistores M2, M4 y M5 a altos valores de intensidad de corriente.

Se puede observar en la figura que prácticamente los puntos arrojan rectas de pendiente similar, lo que estaría confirmando que en estas condiciones el paso de corriente está totalmente controlado por la resistividad del $\mathrm{ZnO}$, pero que aun así, el material conserva la memoria de los efectos barrera observados en las curvas de corriente de fuga, donde se mantenía más elevado el valor de $\mathrm{kV}$ para el M4 que para el M5 y que para el M2.

\begin{tabular}{|c|c|c|c|}
\hline VARISTOR & $\begin{array}{c}\mathrm{d}[\mu \mathrm{m}] \\
\text { Ec. }[2] \text { para } \mathrm{V}_{1 \mathrm{~mA}}\end{array}$ & $\begin{array}{c}d[\mu \mathrm{m}] \\
\text { Ec. }[2] \text { para } V_{5 \mathrm{~mA}}\end{array}$ & $\begin{array}{c}\mathrm{d}[\mu \mathrm{m}] \\
\text { Ec. }[2] \text { para } \mathrm{V}_{10 \mathrm{~mA}}\end{array}$ \\
\hline M1 & 65,4 & 43,5 & 38,7 \\
\hline M2 & 40,2 & 30,7 & 28,2 \\
\hline M3 & 44,6 & 34,0 & 29,9 \\
\hline M4 & 17,9 & 17,3 & 17,1 \\
\hline M5 & 18,9 & 18,0 & 17,8 \\
\hline M6 & 26,8 & 25,1 & 24,5 \\
\hline B & 33,0 & 28,2 & 22,2 \\
\hline
\end{tabular}

Tabla 2.: Distintos valores de tamaño de grano calculados en base a la ecuación [2] para distintos valores de $V$ obtenidos con distintas intensidades de corriente.

Los diferentes varistores en estudio fueron observados por microscopía óptica con magnificaciones entre 40 y 200 aumentos, con y sin nicoles cruzados, con el objeto de detectar macrodefectos e inhomogeneidades.

Se observó que las muestras M1, M2 y M3 poseían un nivel de microfisuras mayor que las muestras de otra procedencia además de una mayor inhomogeneidad y mayor porosidad.

El estudio realizado con microscopia electrónica de barrido y microsonda se ve resumido en las Figuras 5 y 6.

La Figura 5 muestra la comparación entre el varistor M4 (a, b y c) y el varistor M2 ( $\mathrm{a}$, b y c); donde "a" corresponde a una superficie pulida, " $\mathrm{b}$ " a una superficie pulida parcialmente atacada con ácido acético $10 \% \mathrm{v} / \mathrm{v}, \mathrm{y}$ " $\mathrm{c}$ " a una superficie pulida atacada durante $15 \mathrm{~min}$. con una solución ácida como la descripta (Barra de escala $=10 \mu \mathrm{m}$ ).

Comparando los materiales originales (fotos "a") se obser- va que la M2 posee una mayor porosidad residual respecto a la M4. Las fotos " $b$ " muestran que existe una mayor penetración en el ataque parcial de la M2 respecto a la M4 debido principalmente a la mayor porosidad del prototipo, lo que permitió un ataque y penetración del reactivo a distintos niveles de profundidad. Las fotografías " $\mathrm{c}$ " en cambio no muestran mayores diferencias entre sí dado que lo único que muestran ambas son los cristales de $\mathrm{ZnO}$ remanentes habiendo sido eliminada toda la matriz.

La Figura 6 muestra las microfotografías de los materiales M4 y M2 con mayor magnificación (Barra de escala $=10 \mu \mathrm{m}$ ) en donde se pueden observar los bordes de grano. La M2 muestra una menor cantidad de éstos además de la ya mencionada mayor porosidad.

En la parte derecha de la figura se muestra el espectro de energía dispersada, por focalización de la microsonda en la interfase existente entre los granos de $\mathrm{ZnO}$ de cada una de las muestras, observándose que prácticamente no existe antimonio en las zonas analizadas de la muestra prototipo M2, no ocurriendo lo mismo en el varistor comercial M4.

Según Haskell y col. (7) las fases espinela $\left(\mathrm{Zn}_{7} \mathrm{Sb}_{2} \mathrm{O}_{12}\right)$ y pirocloro $\left(\mathrm{Zn}_{2} \mathrm{Bi}_{3} \mathrm{Sb}_{3} \mathrm{O}_{14}\right)$ sirven como puntos de conección para los bordes de grano y de penetración para los otros dopantes del sistema, siendo además el antimonio el generador de la fase espinela que contacta los granos de $\mathrm{ZnO}$; ésta última es la responsable mayoritaria de las propiedades eléctricas del varistor: incrementa del voltaje de ruptura, actúa como agente inhibidor del crecimiento de grano, disminuye el valor de la corriente de fuga y aumenta el coeficiente de no linealiad $(\alpha)$.

Como se ha observado, el prototipo M2 acusa una deficiencia en la cantidad de antimonio en las interfases entre los granos de $\mathrm{ZnO}$, esto está asociado a las no buenas propiedades observadas (tamaño de grano elevado y pobres propiedades en cuanto al efecto varistor), debiendo sumarse a ésto las inhomogeneidades, microfisuras y alta porosidad observadas con la ayuda de la microscopía óptica (defectos de procesamiento).

Es llamativo además el efecto observado en cuanto a la posición del espaciado del plano 311 de la espinela. En el caso de los prototipos (M1, M2 y M3) se observaron mejoras en las propiedades cuanto mayores eran los valores de espaciado, aunque no se han alcanzado los valores que presentaron los varistores comerciales. Probablemente el defecto en antimonio sea el causante de defectos en la estructura de la espinela o de la fase pirocloro, causando una baja en las propiedades globales del varistor.

\section{CONCLUSIONES}

- Se desarrollaron tres prototipos de varistores de $\mathrm{ZnO}$ con distintas composiciones de dopantes.

- Se compararon las características y el comportamiento de éstos con el de otros varistores comerciales.

- Se detectaron fallas de orden textural (procesamiento) y composicional en las interfases de bordes de grano de los prototipos (defecto de antimonio).

- Se determinó valor del espaciado del plano 311 de la fase espinela, sugiriendo que éste puede tener alguna relación con las propiedades finales de los varistores.

- Un mejor control del procesamiento (homogeinización y compactación) y un aumento en el dopaje de $\mathrm{Sb}_{2} \mathrm{O}_{3}$ sobre el prototipo M2 podrían concluír en un varistor de calidad comercial. 

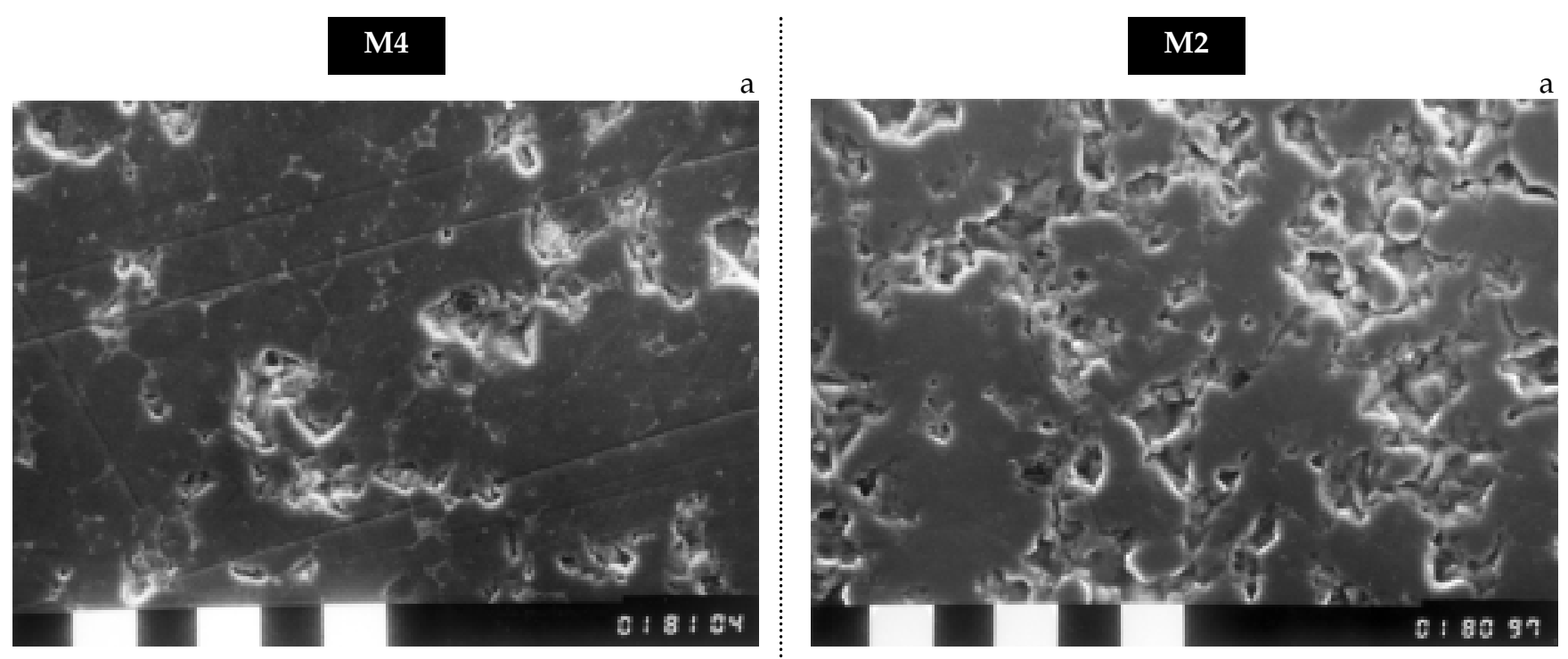

$\mathrm{b}$
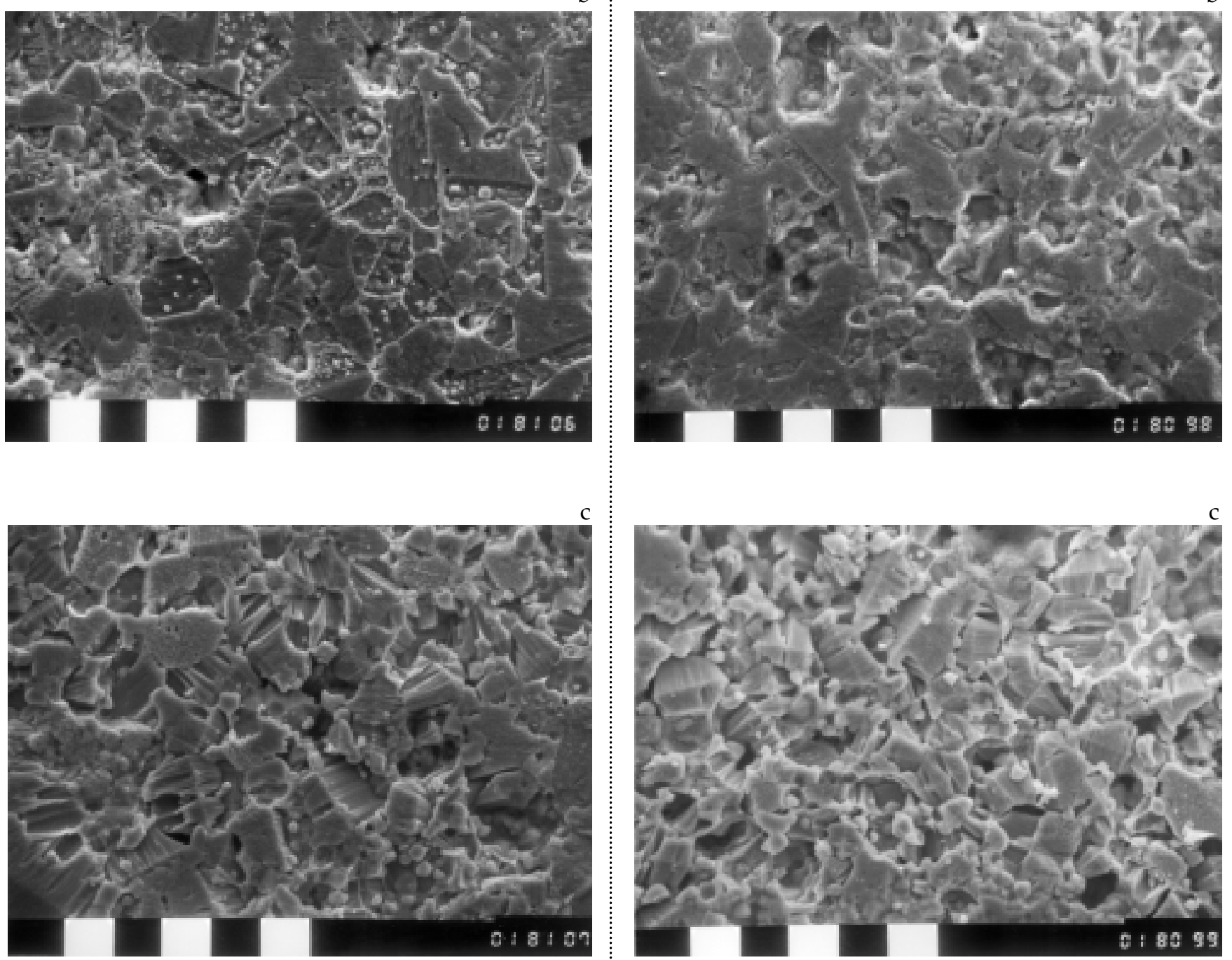

Figura 5. Comparación entre el varistor M4 (a, b y c) y el varistor M2 (a, b y c); donde "a" corresponde a una superficie pulida, " $\mathrm{b}$ " a una superficie pulida parcialmente atacada con ácido acético $10 \% \mathrm{v} / \mathrm{v}, \mathrm{y}$ "c" a una superficie pulida atacada durante 15 min. con una solución ácida como la descripta (Barra de escala $=10 \mu \mathrm{m}$ ). 

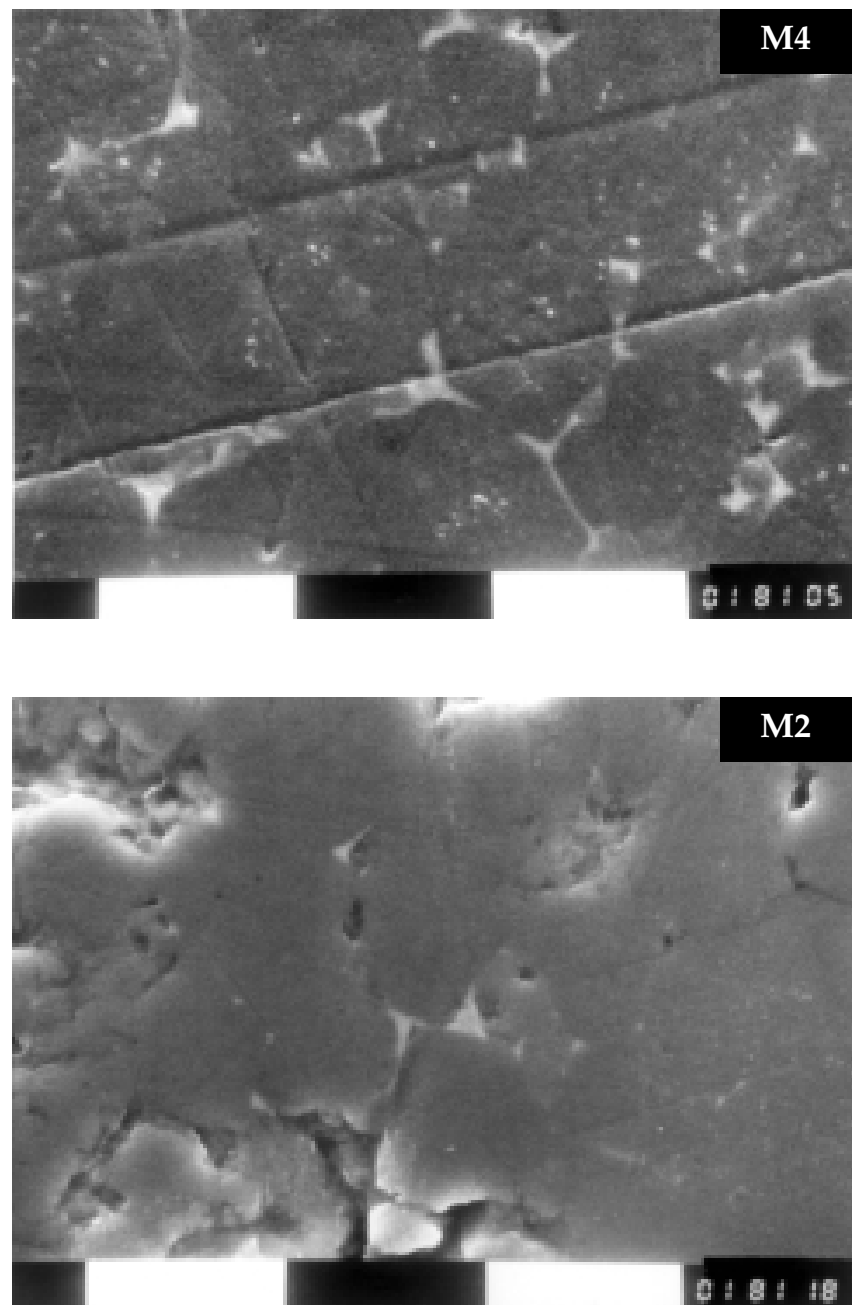

Label A: m41borde

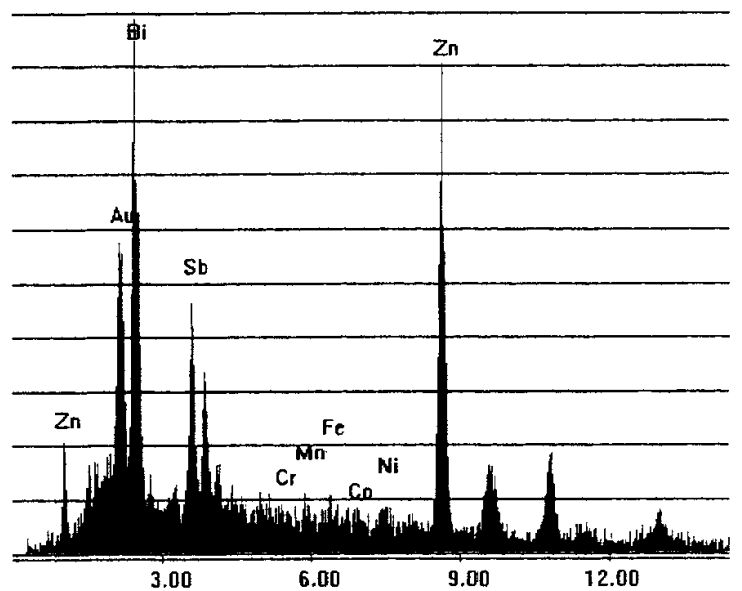

Label A: m21bor2

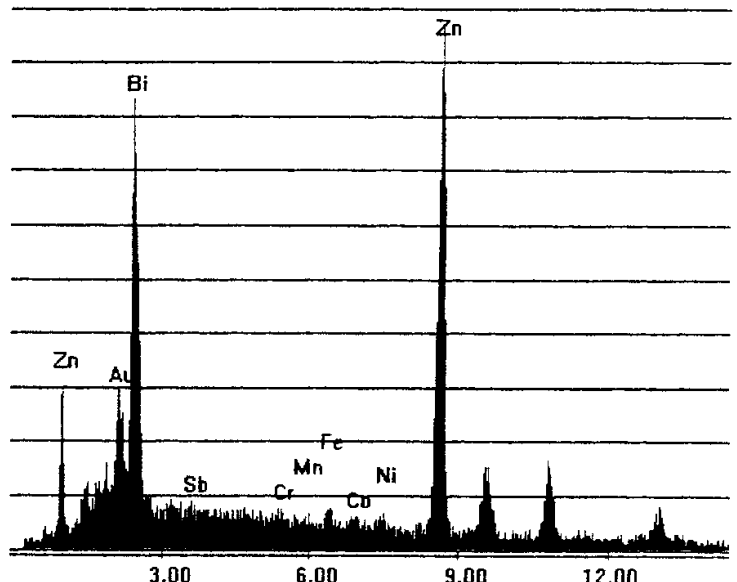

Figura 6. Microfotografías de los materiales M4 y M2 con mayor magnificación (Barra de escala $=10 \mu \mathrm{m}$ )

\section{BIBLIOGRAFÍA}

1. M. Matsuoka, T. Masuyama, Y. Ida. "Non-linear electrical properties of zinc oxide ceramics". Suppl. J. Jpn. Soc. Appl. Phys., 39, 94-101 (1970).

2. M. Matsuoka. "Progress in research and development of zinc oxide varistors". Adv. Ceram., 7, 290-308 (1981)

3. F.D. Martzloff, L.M. Levinson. "Surge-protective devices". In Electronic Ceramics Properties, Devices and Applications, Ed. L. M. Levisnon. Marcel Dekker Inc. N.Y. (1988).

4. L.M. Levinson, H.R. Philip. "Zinc oxide varistors - A review". Am. Cer. Soc.
Bull., 65, 639-46 (1986)

5. A. Lagrange. "Present and future of zinc oxide varistor". In Electronic Ceramics, Ed. by B.C.H. Steele. Elsevier, London (1991).

6. T. Senda, R.C. Bradt. "Grain growth of zinc oxide during the sintering of zinc oxide-antimony oxide ceramics". J. Am. Ceram. Soc., 74, 1296-1302 (1991).

7. B.A. Haskell, S.J. Souri, M.A. Helfand. "Varistor Behavior at Twin Boundaries in $\mathrm{ZnO}^{\prime \prime}$. J. Am. Ceram. Soc. 82 [8], 2106-10 (1999).

\section{Recibido: 01.06 .00}

Aceptado: 02.04.01 